\title{
A Spectrum of Causative Organisms of Healthcare-associated Infections in a Tertiary Care Hospital of West Bengal: An Observational Study
}

\author{
Sinjan Ghosh ${ }^{1}$, Mainak Mukhopadhyay ${ }^{2}$, Anjum Namhata ${ }^{3}$, Nandini Chatterjee ${ }^{4}$
}

\begin{abstract}
Background: Nosocomial infections are the foremost reasons for morbidity and mortality among hospitalized patients. Rampant use of antibiotics in infections has led to the emergence of multiresistant bacteria worldwide. Periodical review of sensitivity profiles is of utmost importance for optimal patient benefit.

Aims and objectives: To explore the spectrum of organisms responsible for intensive care unit (ICU) and inpatient hospital-acquired sepsis and evaluate the pattern of antibiotic sensitivity of the organisms.

Materials and methods: Samples were collected from all consecutive patients getting fever and satisfying the criteria for a nosocomial infection indoor as well as ICU, irrespective of etiology. They included sputum, blood, urine, and wound swabs. All samples were sent for a routine examination as well as a culture and sensitivity test. Descriptive statistical methods were used with the help of SPSS version 21.

Results: The most frequent organisms for nosocomial infections in the ICU were Enterobacteriaceae (45\%), Acinetobacter (13.7\%), and Staphylococcus aureus (12.3\%). In the general wards, the most common isolates were Enterobacteriaceae (59.9\%), S. aureus (14.5\%), Enterococcus (9.8\%), and Pseudomonas (9.3\%). There was a high percentage of extended-spectrum beta-lactamase among the Enterobacteriacae, methicillin-resistant $S$. aureus, and borderline oxacillin-resistant S. aureus among S. aureus and metallo- $\beta$-lactamase among the Acinetobacter and Pseudomonas. These indicate resistance to most beta-lactams, cephalosporins, and at times to carbapenems. There was also coresistance to fluoroquinolones and aminoglycosides. Conclusion: An antibiotic policy should be improvised for each healthcare facility on the basis of that point of time.

Keywords: Infection, Multiresistant, Nosocomial, Sensitivity,

Bengal Physician Journal (2021): 10.5005/jp-journals-10070-7020
\end{abstract}

\section{INTRODUCTION}

Antibiotics are essential components of modern therapeutic practices. But inappropriate and indiscriminate usage of antibiotics can result in resistance of organisms. Healthcare-associated infections (HAls) lead to poor outcomes in hospitalized patients. Thus, it is mandatory to abide by certain norms while prescribing antibiotics to minimize nosocomial infections which are defined as those that occur within 48 hours of admission or 3 days after discharge from a healthcare facility and it was absent during admission. ${ }^{2}$ Empirical antibiotic therapy if not matched with suitable sensitivity profiles definitely leads to polyresistance in severe sepsis. ${ }^{3}$ So, it is essential for the institutions to have in-house up-to-date data to evaluate the likely causatives. This will assist in choosing empirical drugs from sensitivity patterns.

Studies concerning culture and sensitivity profiles of body fluids have been performed over the decades. There have been changes in the sensitivity profiles over time and more so due to the indiscriminate as well as illogical use of antibiotics. We endeavored to study the current trend of causative organisms and their antibiotic sensitivity and to compare it with the previous work done in this field. This study was done for 6 months to identify the pattern of organisms isolated from the intensive care unit (ICU) and inpatients of wards.

\section{Aims and Objectives}

- Primary objective is to explore the spectrum of organisms responsible for inpatient nosocomial sepsis.

- Secondary objective is to evaluate the pattern of antibiotic sensitivity in the organisms.
${ }^{1}$ Department of Neurology, NRS Medical College, Kolkata, West Bengal, India

${ }^{2}$ Department of Cardiology, Midnapur Medical College Hospital, Midnapur, West Bengal, India

${ }^{3}$ Department of Microbiology, NRS Medical College, Kolkata, West Bengal, India

${ }^{4}$ Department of Medicine, IPGMER and SSKM Hospital, Kolkata, West Bengal, India

Corresponding Author: Anjum Nanhata, Department of Microbiology, NRS Medical College, Kolkata, West Bengal, India, Phone: +918902221317, e-mail: rivuc23092006@gmail.com

How to cite this article: Ghosh S, Mukhopadhyay M, Namhata A, et al. A Spectrum of Causative Organisms of Healthcare-associated Infections in a Tertiary Care Hospital of West Bengal: An Observational Study. Bengal Physician Journal 2020;7(1):2-7.

Source of support: Nil

Conflict of interest: None

\section{Materials and Methods}

This is an observational study carried out in the ICU and the general wards of our hospital for a period of 6 months. Nonpurposive convenient sampling method was used to select patients. All patients with undiagnosed fever that developed 48 hours after admission to the hospital were included in the study fulfilling the criteria for a nosocomial infection. Immunocompromised and neutropenic patients and patients on steroids or immunosuppressive drugs

(c) The Author(s). 2020 Open Access This article is distributed under the terms of the Creative Commons Attribution 4.0 International License (https:// creativecommons.org/licenses/by-nc/4.0/), which permits unrestricted use, distribution, and non-commercial reproduction in any medium, provided you give appropriate credit to the original author(s) and the source, provide a link to the Creative Commons license, and indicate if changes were made. The Creative Commons Public Domain Dedication waiver (http://creativecommons.org/publicdomain/zero/1.0/) applies to the data made available in this article, unless otherwise stated. 
were excluded. After taking informed consent, detailed history and clinical examination were recorded in a prevalidated proforma.

Antimicrobial sensitivity tests were performed on bacteria considered significant. The antibiotics included in our study were Co-amoxiclav, ampicillin, trimethoprim/sulfamethoxazole (cotrimoxazole), norfloxacin, ciprofloxacin, levofloxacin, ofloxacin, nitrofurantoin, gentamicin, amikacin, doxycycline, cefuroxime, ceftazidime, cefotaxime, netilmicin, polymyxin B, Colistin, piperacillin/tazobactam, cefoperazone/sulbactam, meropenem, imipenem, vancomycin, tigecycline, and teicoplanin.

\section{Results}

The study was conducted for 6 months in the general wards as well as ICU of a tertiary care hospital. The total number of samples collected was 1,046, of which 255 were from ICU. Seventy-three were positive isolates in the ICU (Table 1). Of 73 isolates, respiratory tract infection accounted for 33, urinary tract infection (UTI) 10, wound sepsis 7, bacteremia/septicemia 11, and intravenous (IV) catheter sepsis in 2 patients. Ten samples (15.4\%) were due to probable colonization.

Table 1: Antibiotic sensitivity patterns of common isolates from inpatients location: intensive care units

\begin{tabular}{|c|c|c|c|c|c|}
\hline \multirow[b]{3}{*}{ Antibiotics } & \multicolumn{5}{|c|}{ Isolates (\% sensitive) } \\
\hline & Enterobacteriaceae & Pseudomonas & Acinetobacter & Staphylococci & Enterococci \\
\hline & $N=34$ & $N=9$ & $N=10$ & $N=9$ & $N=5$ \\
\hline Amoxicillin & 00 & - & - & 11 & 60 \\
\hline Cloxacillin/oxacillin & - & - & - & 44 & - \\
\hline Carbenicillin & - & 00 & - & - & - \\
\hline Piperacillin & - & 00 & - & - & - \\
\hline Ampicillin-sulbactam & - & - & 60 & - & - \\
\hline Amoxicillin-clavulanate & 06 & - & 10 & 11 & 60 \\
\hline Piperacillin-tazobactam & 62 & 44 & 80 & 56 & 100 \\
\hline Ticarcillin-clavulanate & 35 & 00 & 20 & 56 & - \\
\hline Cefoperazone-sulbactam & 65 & 56 & 100 & 56 & - \\
\hline Cephalexin & - & - & - & 56 & - \\
\hline Cefuroxime & 16 & - & - & 56 & - \\
\hline Cefotaxime & 24 & - & 20 & - & - \\
\hline Ceftazidime & 24 & 11 & 20 & - & - \\
\hline Cefoperazone & - & 11 & - & - & - \\
\hline Cefpirome & - & - & - & 56 & - \\
\hline Cefepime & 24 & 11 & 20 & - & - \\
\hline Aztreonam & 24 & 11 & 20 & - & - \\
\hline Imipenem & 100 & 78 & 60 & - & 100 \\
\hline Meropenem & 100 & 78 & 60 & - & - \\
\hline Azithromycin & - & - & - & 33 & 40 \\
\hline Clindamycin & - & - & - & 67 & - \\
\hline Vancomycin & - & - & - & 100 & 100 \\
\hline Teicoplanin & - & - & - & 100 & 100 \\
\hline Linezolid & - & - & - & 100 & 100 \\
\hline Gentamicin & 56 & 22 & 70 & 89 & (High dose) 60 \\
\hline Tobramycin & 47 & 22 & 80 & - & - \\
\hline Netilmicin & 68 & 67 & 80 & 100 & 60 \\
\hline Amikacin & 65 & 67 & 80 & 100 & 60 \\
\hline Ciprofloxacin & 35 & 44 & 40 & 56 & 20 \\
\hline Ofloxacin & 38 & 44 & 60 & 56 & 20 \\
\hline Levofloxacin & 62 & 44 & 80 & 56 & 20 \\
\hline Moxifloxacin & 56 & 44 & 80 & 78 & 60 \\
\hline Cotrimoxazole & 21 & 00 & 40 & 33 & - \\
\hline Chloramphenicol & 68 & - & 40 & 78 & 40 \\
\hline Tetracycline & 44 & - & 50 & 89 & 20 \\
\hline Tigecycline & 94 & - & 100 & 100 & 80 \\
\hline Polymyxin B & - & 89 & 100 & - & - \\
\hline Colistin & - & 89 & 100 & - & - \\
\hline
\end{tabular}

Total no. of samples: 1464; no. of inpatient samples: 1046; no. of ICU samples: 255 ; total no. of ICU isolates: 73 ; polymicrobial growth: 8 samples (12.3\%); NA, antibiotic disks not available 


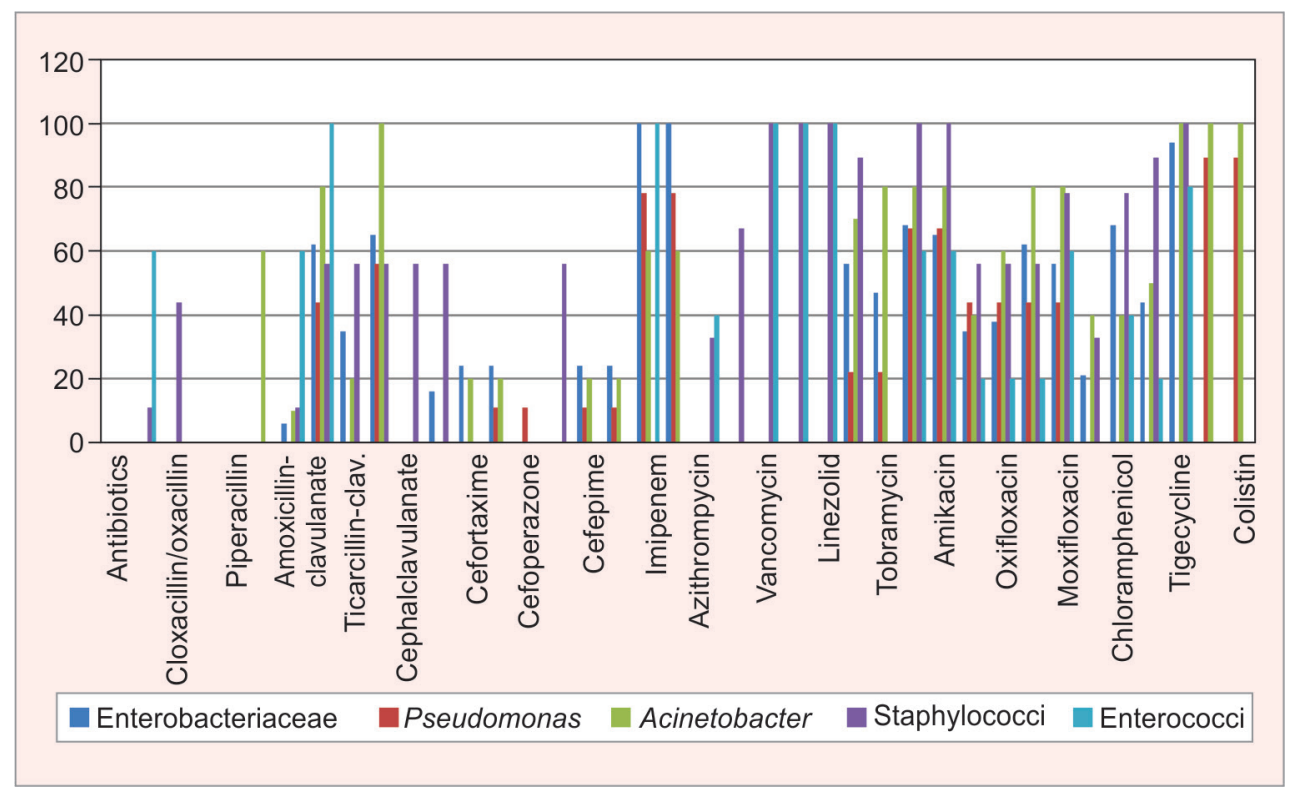

Fig. 1: Sensitivity patterns of organisms in ICU

The most common organisms isolated were Enterobacteriaceae (34 isolates, i.e., 45\%). Of which 15 (44\%) were extended-spectrum beta-lactamase (ESBL) producers; 11 (32\%) probable coproducers of ESBLs and AmpC beta-lactamases. All isolates were sensitive to imipenem and meropenem.

Among the Acinetobacter sp., the next most common organism (10 isolates, i.e., 13.7\%), 4 (40\%) were carbapenem resistant and 3 (75\%) of those were metallo- $\beta$-lactamase (MBL) producers. Polymyxins, cefoperazone/sulbactam and tigecycline covered $100 \%$ of the isolates. Other organisms isolated were:

- Staphylococci: 9 isolates (12.3\%), of which 3 were (33\%) methicillin-resistant S. aureus (MRSA), 1 (11\%) borderline oxacillin-resistant $S$. aureus (BORSA), 4 methicillin- sensitive $S$. aureus (MSSA), 1 methicillin-resistant coagulase-negative $S$. aureus (MR-CNSA).

- Pseudomonas: 9 isolates (12.3\%), of which 2 (22\%) were carbapenem-resistant MBL producers. All Pseudomonas aeruginosa isolates were sensitive to polymyxins.

- Enterococci: 5 isolates (6.8\%): all Enterococcus faecalis: all were sensitive to vancomycin, linezolid, imipenem, and piperacillin/ tazobactam (Fig. 1).

- Fungi: 6 isolates (8.2\%): all Candida sp.: 4 (67\%) albicans; 2 (33\%) non-albicans spp.

The results from inpatients revealed the following (Table 2): there were 791 samples collected, of which 172 were positive. Of 172 isolates, respiratory tract infection accounted for 10, UTI 70, wound sepsis 60, bacteremia/septicemia 15, IV catheter infection in 3; 14 samples (9\%) were due to probable colonization.

Here also the most frequent isolate was Enterobacteriaceae: 103 isolates (59.9\%). Fifty-four (52\%) ESBL producers; 11 (11\%) probable coproducers of ESBLs and AmpC beta-lactamases. All were carbapenem sensitive. They were poorly responsive to cephalosporins and quinolones.

Next were Staphylococci: 25 isolates (14.5\%): 10 (40\%) MRSA, 2 (8\%) BORSA, 13 MSSA. Well responsive to vancomycin, clindamycin, and linezolid.
Enterococci: Of the 17 isolates (9.8\%), all were sensitive to vancomycin, linezolid, and tigecycline.

Pseudomonas: 16 isolates (9.3\%): all P. aeruginosa: 6 (38\%) carbapenem resistant: all were MBL producers. All were polymyxin sensitive.

Acinetobacter sp.: 3 isolates (1.7\%): 1 (33\%) carbapenem resistant and MBL producer. All sensitive to polymyxins, cefoperazone/ sulbactam, and piperacillin/tazobactam (Fig. 2).

Fungi: 8 isolates (4.6\%), all Candida sp.: 4 (50\%) albicans; 4 (50\%) non-albicans spp.

To summarize, the pattern of infection reveals a spectrum of multiresistant bacteria both in ICU and general wards responsive to the highest grade of antibiotics. However, Acinetobacter was much more frequently found in ICU than wards, though Enterobacteriacae and S. aureus were omnipresent. ${ }^{4}$

\section{Discussion}

HAls comprise catheter-associated UTI, pneumonia including ventilator-associated pneumonia, bloodstream infection, gastroenteritis, and surgical site and wound sepsis. ${ }^{5}$ Nosocomial infections are most frequently bacterial. ${ }^{6}$ Some may be commensals of the patient taking the upper hand when immunity becomes weak. In the ICU, almost $70 \%$ of the organisms constitute Staphylococci, Enterobacteriaceae, Pseudomonas, and Acinetobacter species. Enterobacteriaceae include Escherichia coli, Proteus mirabilis, Klebsiella_pneumoniae, and Serratia marcescens. ${ }^{7-9}$

Our study revealed that growth of Enterobacteriaceae, Acinetobacter, S. aureus, and Pseudomonas were most isolated in the ICU. In the wards, Enterobacteriacae, S. aureus, and Pseudomonas were most frequent. This spectrum is similar to the studies in the United Kingdom, Canada, Uganda, etc. ${ }^{10-12}$ Of the Enterobacteriaceae 103 isolates: 54 (52\%) ESBL producers; 11 (11\%) probable coproducers of ESBLs and AmpC beta-lactamases. Among the Staphylococci 25 isolates: 10 (40\%) MRSA; 2 (8\%) BORSA; 13 MSSA. For Acinetobacter sp. 3 isolates: 1 (33\%) carbapenem resistant and MBL producer and 
A Spectrum of Causative Organisms of Healthcare-associated Infections

Table 2: Antibiotic sensitivity patterns of common isolates from inpatients, location: general wards (non-ICU)

\begin{tabular}{|c|c|c|c|c|c|}
\hline \multirow[b]{3}{*}{ Antibiotics } & \multicolumn{5}{|c|}{ Isolates (\% sensitive) } \\
\hline & Enterobacteriaceae & Pseudomonads & Acinetobacter & Staphylococci & Enterococci \\
\hline & $N=103$ & $N=16$ & $N=3$ & $N=25$ & $N=17$ \\
\hline Amoxicillin & 06 & - & - & 16 & 65 \\
\hline Cloxacillin/oxacillin & - & - & - & 52 & - \\
\hline Carbenicillin & - & 13 & - & - & - \\
\hline Piperacillin & - & 13 & - & - & - \\
\hline Ampicillin-sulbactam & - & - & 67 & - & - \\
\hline Amoxicillin-clavulanate & 17 & - & 00 & 24 & 65 \\
\hline Piperacillin-tazobactam & 87 & 50 & 100 & 60 & 82 \\
\hline Ticarcillin-clavulanate & 41 & 31 & 00 & 60 & - \\
\hline Cefoperazone-sulbactam & 88 & 50 & 100 & 60 & - \\
\hline Cephalexin & - & - & - & 60 & - \\
\hline Cefuroxime & 20 & - & - & 60 & - \\
\hline Cefotaxime & 32 & - & 00 & - & - \\
\hline Ceftazidime & 32 & 19 & 00 & - & - \\
\hline Cefoperazone & - & 19 & - & - & - \\
\hline Cefpirome & - & - & - & - & - \\
\hline Cefepime & 32 & 25 & 00 & - & - \\
\hline Aztreonam & 32 & 38 & 00 & - & - \\
\hline Imipenem & 100 & 63 & 67 & - & 82 \\
\hline Meropenem & 100 & 63 & 67 & - & - \\
\hline Azithromycin & - & - & - & 56 & 47 \\
\hline Clindamycin & - & - & - & 68 & - \\
\hline Vancomycin & - & - & - & 100 & 100 \\
\hline Teicoplanin & - & - & - & 100 & 100 \\
\hline Linezolid & - & - & - & 100 & 100 \\
\hline Gentamicin & 61 & 50 & 33 & 92 & (High dose) 59 \\
\hline Tobramycin & 59 & 50 & 33 & - & - \\
\hline Netilmicin & 71 & 50 & 67 & 92 & 65 \\
\hline Amikacin & 70 & 69 & 33 & 92 & 59 \\
\hline Ciprofloxacin & 41 & 50 & 33 & 68 & 35 \\
\hline Ofloxacin & 47 & 50 & 33 & 76 & 47 \\
\hline Levofloxacin & 69 & 50 & 67 & 88 & 59 \\
\hline Moxifloxacin & 83 & 50 & 67 & 92 & 76 \\
\hline Cotrimoxazole & 34 & 19 & 33 & 44 & - \\
\hline Chloramphenicol & 68 & - & - & 88 & 53 \\
\hline Tetracycline & 54 & - & 33 & 92 & 47 \\
\hline Tigecycline & 95 & - & 67 & 100 & 100 \\
\hline Polymyxin B & - & 100 & 100 & - & - \\
\hline Colistin & - & 100 & 100 & - & - \\
\hline
\end{tabular}

No. of inpatient samples: 1046; no. of non-ICU samples: 791; total no. of non-ICU isolates: 172; polymicrobial growth: 15 samples (9.6\%); NA, antibiotic discs not available

for Pseudomonas 6 isolates (38\%) carbapenem resistant: all were MBL producers. A study from Punjab revealed production of ESBL, AmpC beta-lactamase, and MBL by E. coli and K. pneumoniae.

Of all isolates, ESBL was detected in $35.16 \%$ and MBL producers in $10.98 \%$. $^{13}$

Thus, they manifested multidrug resistance and propensity to respond only to higher antibiotics like carbapenems, polymyxin, Colistin, vancomycin, tigecycline, etc. The initial choice of drugs like cephalosporins and beta-lactams is useless in this setting.
Coresistance to quinolones and aminoglycosides was seen maybe due to plasmid transfer, which is of grave concern.

The fungal isolate in this study, i.e., Candida species is another fixture in all HAl spectrum of organisms. It is isolated mostly in patients with diabetes, renal failure, and cancer and in immunocompromised patients. It is said to be the sixth common cause of nosocomial infection worldwide. ${ }^{14}$

World Health Organization documents around $15 \%$ of all hospitalized patients develop nosocomial infections. ${ }^{15}$ Susceptibility 


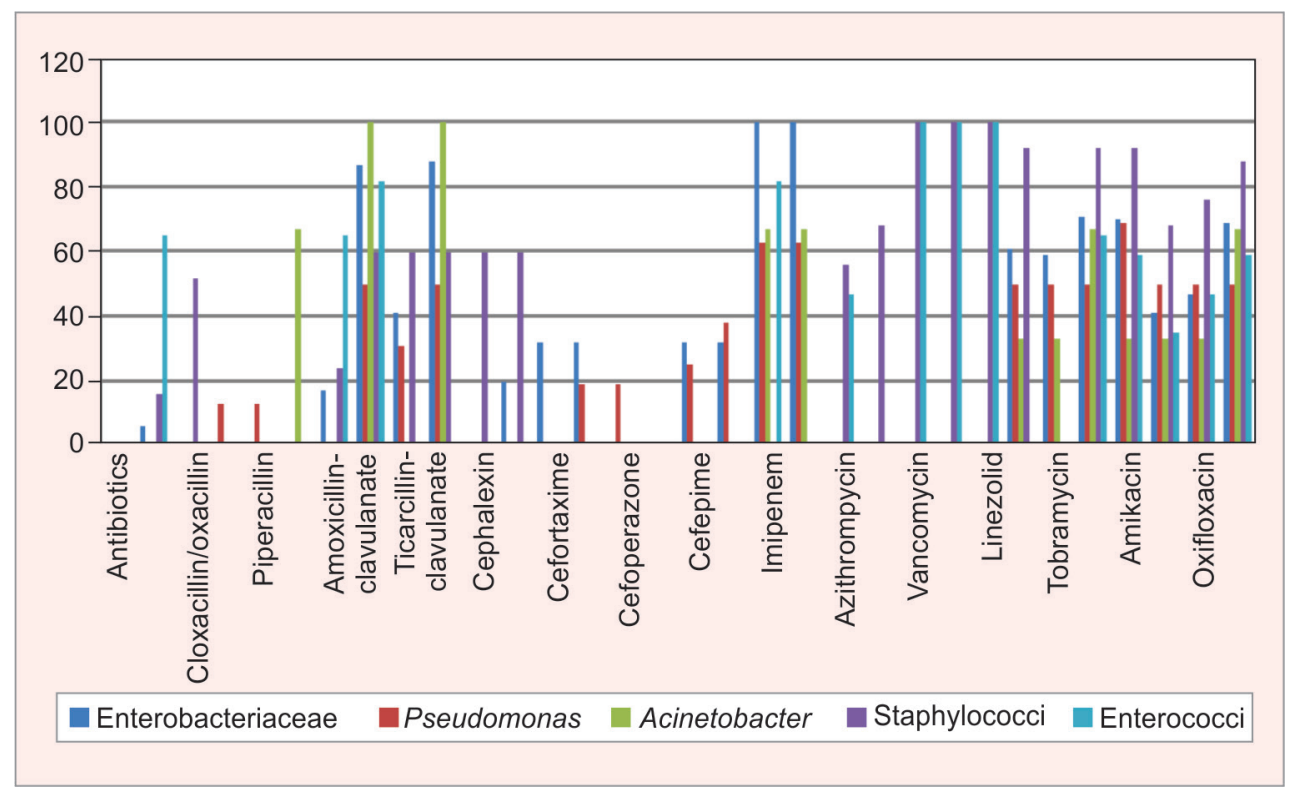

Fig. 2: Sensitivity patterns of organisms in wards

to $\mathrm{HAl}$ increases due to unhygienic environment, improper waste disposal, and ignorance among healthcare personnel. ${ }^{16}$

Patient factors include immunosupression, increased duration of ICU admission, and continued antibiotic therapy. Improper techniques of use of invasive devices (cannula and catheters) and absence of control policies are all incriminated as predisposing factors. ${ }^{17}$

In low-income countries, the above factors are linked to poor economic status, inadequate staff, and infrastructure. ${ }^{18}$

It is important to devise ways of prevention of nosocomial infections by practicing well-monitored healthcare delivery, maintenance of hygiene, and specifically targeted antimicrobial prescription policy.

Proper waste disposal, cleanliness in the environment, proper food handling, and hand hygiene should be practiced. Foremost is also to take measures for the prevention of drug resistance to develop. ${ }^{19,20}$

It is important that every hospital should have an infection control group who would devise an antibiotic policy for that particular healthcare facility. Our study is a typical microbiological surveillance report on the current pathogen spectrum and their sensitivity pattern.

An antibiotic policy provides guidelines for use in specific infections for that institution at a point in time. It is reviewed by a microbiologist and devised based on the latest surveillance data. ${ }^{21,22}$

The following are the measures to be undertaken:

a. Training on antibiotic usage.

b. Monthly data surveillance and feedback to be reviewed periodically.

c. Hospital infection control group will formulate guidelines to ensure restricted antibiotic use.

\section{Limitations of OUR Study}

Viral identification was not possible in our setup. The total number of samples was limited and stool samples were not done.

\section{Conclusion}

Superbugs are emerging day by day in healthcare setups where traditional antibiotics are becoming useless. The importance of periodical sampling and culture and sensitivity tests cannot be undermined as the bacterial spectrum and the sensitivity change over time. Moreover, it helps to lay down an antibiotic policy for a particular institution that goes a long way in the prevention of multidrug resistance in the future.

\section{References}

1. Khan HA, Baig FK, Mehboob R. Nosocomial infections: epidemiology, prevention, control and surveillance. Asian Pac J Trop Biomed 2017;7(5): 478-482. DOI: 10.1016/j.apjtb.2017.01.019.

2. Gupta A, Singh DK, Krutarth, B, et al. Prevalence of health care associated infections in a tertiary care hospital in Dakshina Kannada, Karnataka: a hospital based cross sectional study. Int J Med Res Health Sci 2015;4(2):317-321. DOI: 10.5958/2319-5886.2015.00059.4.

3. Lee $\mathrm{C}-\mathrm{C}$, Lee $\mathrm{C}-\mathrm{H}$, Chuang $\mathrm{M}-\mathrm{C}$, et al. Impact of inappropriate empirical antibiotic therapy on outcome of bacteremic adults visiting the ED. Am J Emerg Med 2012;30(8):1447-1456. DOI: 10.1016/j. ajem.2011.11.010.

4. Emily RM, Sydnor TMP. Hospital epidemiology and infection control in acute-care settings. Clin Microbiol Rev 2011;24(1):141-173. DOI: 10.1128/CMR.00027-10.

5. Raja Danasekaran GM, Annadurai K. Prevention of healthcareassociated infections: protecting patients, saving lives. Int J Commun Med Publ Health 2014;1(1):67-68. DOI: 10.5455/2394-6040. ijcmph20141114.

6. Khan H, Ahmad A, Mehboob R. Nosocomial infections and their control strategies. Asian Pac J Trop Biomed 2015;5(7):509-514. DOI: 10.1016/j.apjtb.2017.01.019

7. Brasme L, Nordmann P, Fidel F, et al. Incidence of class A extendedspectrum beta-lactamases in Champagne-Ardenne (France): a 1 year prospective study. J Antimicrob Chemother 2007;60(5):956-964. DOI: 10.1093/jac/dkm319.

8. Eggimann P, Pittet D. Infection control in the ICU. Chest 2001;120(6):2059-2093. DOI: 10.1378/chest.120.6.2059.

9. Weinstein RA. Nosocomial infection update. Emerg Infect Dis 1998;4(3):416-420. DOI: 10.3201/eid0403.980320. 
10. Ojulong J, Mwambu TP, Joloba M, et al. Relative prevalence of methicilline resistant Staphylococcus aureus and its susceptibility pattern in Mulago Hospital, Kampala, Uganda. Tanzan J Health Res. 2009;11(3):149-153. DOI: 10.4314/thrb.v11i3.47703.

11. Chambers HF. Community-associated MRSA-resistance and virulence converge. N Engl J Med 2005;352(14):1485-1487. DOI: 10.1056/ NEJMe058023.

12. Zhanel GG, DeCorby M, Laing N, et al. Antimicrobial-resistant pathogens in intensive care units in Canada: results of the Canadian National Intensive Care Unit (CAN-ICU) study, 2005-2006. Antimicrob Agents Chemother 2008;52(4):1430-1437. DOI: 10.1128/AAC.01538-07.

13. Oberoi L, Singh N, Sharma P, et al. ESBL, MBL and Ampc $\beta$ lactamases producing superbugs - Havoc in the intensive care units of Punjab India. J Clin Diagnostic Res 2013;7(1):70-73. DOI: 10.7860/ JCDR/2012/5016.2673.

14. Safdar N, Maki DG. The commonality of risk factors for nosocomial colonization and infection with antimicrobial-resistant Staphylococcus aureus, Enterococcus, gram-negative bacilli, Clostridium difficile, and Candida. Ann Intern Med 2002;136(11):834-844. DOI: 10.7326/00034819-136-11-200206040-00013.

15. Nejad SB, Syed SB, Ellis B, et al. Health-care-associated infection in Africa: a systematic review. Bull World Health Org. 2011;89:757-765. DOI: 10.2471/BLT.11.088179.
16. Inci A, Karabay A, Erus S, et al. Nosocomial infections and associated risk factors in geriatric patients in the intensive care unit. Eurasian $J$ Emerg Med 2016;15:177-180. DOI: 10.5152/eajem.2016.35744.

17. Ozdemir K, Dizbay M, Dikmen A. Incidence and risk factors of nazocomial infections in elderly and adult patients in intensive care units. Turk J Geriatr 2013;16:155-160. DOI: 10.5799/ ahinjs.02.2015.01.0174.

18. Bardossy AC, Zervos J, Zervos M. Preventing hospital-acquired infections in low-income and middle-income countries: impact, gaps, and opportunities. Infect Dis Clin North Am 2016;30(3):805-818. DOI: 10.1016/j.idc.2016.04.006.

19. Blot S. Limiting the attributable mortality of nosocomial infection and multidrug resistance in intensive care units. Clin Microbiol Infect 2008;14(1):5-13. DOI: 10.1111/j.1469-0691.2007.01835.x.

20. Allegranzi B, Pittet $D$. Role of hand hygiene in healthcare-associated infection prevention. J Hosp Infect 2009;73(4):305-315. DOI: 10.1016/j. jhin.2009.04.019.

21. Jarvis WR. Infection control and changing health-care delivery systems. Emerg Infect Dis 2001;7(2):170-173. DOI: 10.3201/eid0702.010202.

22. Haley RW, Culver DH, White JW, et al. The efficacy of infection surveillance and control programs in preventing nosocomial infections in US hospitals. Am J Epidemiol 1985;121(2):182-205. DOI: 10.1093/oxfordjournals.aje.a113990. 